OPEN ACCESS

Edited by:

Axel Montagne,

University of Edinburgh,

United Kingdom

Reviewed by:

Giuseppe Barisano,

University of Southern California,

United States

Elena Zenaro,

University of Verona, Italy

${ }^{*}$ Correspondence:

Jaime Ramos-Cejudo

Jaime.ramoscejudo@nyulangone.org

Ricardo S. Osorio

Ricardo.Osorio@nyulangone.org

${ }^{\dagger}$ These authors have contributed equally to this work

Received: 10 September 2021 Accepted: 19 October 2021 Published: 30 November 2021

Citation:

Ramos-Cejudo J, Johnson AD, Beiser A, Seshadri S, Salinas J,

Berger JS, Fillmore NR, Do N, Zheng C, Kovbasyuk Z, Ardekani BA,

Bubu OM, Parekh A, Convit A,

Betensky RA, Wisniewski TM and Osorio RS (2021) The Neutrophil

to Lymphocyte Ratio Is Associated

With the Risk of Subsequent

Dementia in the Framingham Heart

Study.

Front. Aging Neurosci. 13:773984.

doi: 10.3389/fnagi.2021.773984

\section{The Neutrophil to Lymphocyte Ratio Is Associated With the Risk of Subsequent Dementia in the Framingham Heart Study}

\author{
Jaime Ramos-Cejudo ${ }^{1,2 *}$, Andrew D. Johnson ${ }^{3,4}$, Alexa Beiser ${ }^{4,5,6}$, Sudha Seshadri4,6,7, \\ Joel Salinas ${ }^{4,8}$, Jeffrey S. Berger ${ }^{9,10,11}$, Nathanael R. Fillmore 2,12 , Nhan Do ${ }^{2,13}$, \\ Chunlei Zheng', Zanetta Kovbasyuk', Babak A. Ardekani1,14, Omonigho M. Bubu', \\ Ankit Parekh' ${ }^{15}$, Antonio Convitt ${ }^{1,14}$, Rebecca A. Betensky ${ }^{16}$, Thomas M. Wisniewski ${ }^{1,8,17}$ \\ and Ricardo S. Osorio ${ }^{1,14 *+}$
}

\begin{abstract}
${ }^{1}$ Department of Psychiatry, New York University Grossman School of Medicine, New York, NY, United States, ${ }^{2}$ VA Boston Cooperative Studies Program, MAVERIC, VA Boston Healthcare System, Boston, MA, United States, ${ }^{3}$ Population Sciences Branch, Division of Intramural Research, National Heart, Lung, and Blood Institute, Framingham, MA, United States, ${ }^{4}$ The Framingham Heart Study, Boston, MA, United States, ${ }^{5}$ Department of Biostatistics, Boston University School of Public Health, Boston, MA, United States, ${ }^{6}$ Department of Neurology, Boston University School of Medicine, Boston, MA, United States, ${ }^{7}$ Glenn Biggs Institute for Alzheimer's and Neurodegenerative Diseases, University of Texas Health Science Center at San Antonio, San Antonio, TX, United States, ${ }^{8}$ Department of Neurology, Center for Cognitive Neurology, NYU Grossman School of Medicine, New York, NY, United States, ${ }^{9}$ Division of Vascular Surgery, Department of Surgery, NYU Grossman School of Medicine, New York, NY, United States, ${ }^{10}$ Division of Cardiology and Hematology, Department of Medicine, NYU Grossman School of Medicine, New York, NY, United States, ${ }^{11}$ Center for the Prevention of Cardiovascular Disease, NYU Grossman School of Medicine, New York, NY, United States, ${ }^{12}$ Harvard Medical School, Boston, MA, United States, ${ }^{13}$ Boston University School of Medicine, Boston, MA, United States, ${ }^{14}$ Nathan Kline Institute, Orangeburg, NY, United States, ${ }^{15}$ Division of Pulmonary, Critical Care and Sleep Medicine, Icahn School of Medicine at Mount Sinai, New York, NY, United States, ${ }^{16}$ Department of Biostatistics, New York University School of Global Public Health, New York, NY, United States, ${ }^{17}$ Department of Pathology, NYU Grossman School of Medicine, New York, NY, United States
\end{abstract}

Objective: Active neutrophils are important contributors to Alzheimer's disease (AD) pathology through the formation of capillary stalls that compromise cerebral blood flow (CBF) and through aberrant neutrophil signaling that advances disease progression. The neutrophil to lymphocyte ratio (NLR) is a proxy of neutrophil-mediated inflammation, and higher NLR is found in persons diagnosed with clinical AD. The objective of this study was to investigate whether increased NLR in older adults is independently associated with the risk of subsequent dementia.

Methods: We examined associations of baseline NLR with incident dementia risk in the community-based Framingham Heart Study (FHS) longitudinal cohorts. The association between NLR and risk of dementia was evaluated using the cumulative incidence function (CIF) and inverse probability-weighted Cox proportional cause-specific hazards regression models, with adjustment for age, sex, body mass index (BMI), systolic and diastolic blood pressure, diabetes, current smoking status, low-density lipoprotein 
$(\mathrm{LDH})$, high-density lipoprotein ( $\mathrm{LDL})$, total cholesterol, triglycerides, and history of cardiovascular disease (CVD). Random forest survival models were used to evaluate the relative predictive value of the model covariates on dementia risk.

Results: The final study sample included 1,648 participants with FHS (average age, 69 years; 56\% women). During follow-up (median, 5.9 years), we observed 51 cases of incident dementia, of which 41 were $A D$ cases. Results from weighted models suggested that the NLR was independently associated with incident dementia, and it was preceded in predictive value only by age, history of CVD, and blood pressure at baseline.

Conclusion: Our study shows that individuals with higher NLR are at a greater risk of subsequent dementia during a 5.9-year follow-up period. Further evaluating the role of neutrophil-mediated inflammation in AD progression may be warranted.

Keywords: Alzheimer's disease, dementia, Framingham, FHS, neutrophil to lymphocyte ratio, NLR, complete blood count (CBC), risk prediction

\section{INTRODUCTION}

The variability of amyloid-beta $(\mathrm{A} \beta)$ and tau pathology associated with clinical symptoms reflects that additional factors influence the progression of cognitive decline in Alzheimer's disease $(\mathrm{AD})$. Vascular dysfunction and inflammation have been implicated in the pathogenesis of $\mathrm{AD}$ in the earliest stages of the disease prior to significant $A \beta$ and tau accumulation (Iturria-Medina et al., 2016; Love and Miners, 2016; Nortley et al., 2019). In addition, clinical studies have indicated that the inflammatory status influences the rate of disease progression in patients with AD (Holmes et al., 2009; Cunningham and Hennessy, 2015).

Recently, neutrophils have been identified as potential key elements of innate immunity contribution to the disease, and a hyperactive neutrophil state has been found in patients with $\mathrm{AD}$ and has been associated with clinical progression (Dong et al., 2018). Neutrophils are the most abundant leukocyte type in the human blood, and although the best characterized function of neutrophils in the defense against infectious pathogens, neutrophils are implicated in the repair of both infectious and sterile injuries (Kruger et al., 2015). Results in animal models of $\mathrm{AD}$ have suggested that neutrophils may be implicated in the breakdown of the blood-brain barrier (BBB) and recruited in the brain parenchyma through the integrin LFA-1 predominantly in perivascular regions with $A \beta$ deposition (Baik et al., 2014; Zenaro et al., 2015). A potential role of neutrophils in the hyperphosphorylation of tau has also been suggested (Zenaro et al., 2015; Nemeth et al., 2020).

Neutrophils are known to have a very short life cycle ranging from just a few hours to up to 5-6 days in circulation (Pillay et al., 2010). During normal inflammation, neutrophils are cleared

Abbreviations: AD, Alzheimer's disease; NLR, neutrophil to lymphocyte ratio; CI, confidence interval; CVD, cardiovascular disease; DSM-IV, Diagnostic and Statistical Manual of Mental Disorders, 4th edition; FHS, Framingham Heart Study; HR, hazard ratio; MMSE, Mini-Mental State Examination; CBC, complete blood count. from the circulation once the initial inflammatory insult is resolved. In aging, however, inaccurate neutrophil chemotaxis may lead to compromised clearance of neutrophils, potentially contributing to a chronic inflammation state with reduced formation of neutrophil extracellular traps (NET), phagocytic response, and reactive oxygen species (ROS) production (Tseng and Liu, 2014). These abnormalities may partially explain the contribution of neutrophil adhesion to brain capillaries in the formation of capillary stalls and reduction in cerebral blood flow (CBF) that is observed in animal models (Cruz Hernandez et al., 2019; El Amki et al., 2020), a mechanism that could occur at the early stages in aging and influence the pathological accumulation of $A \beta$ and tau when sustained over years.

One of the most widely studied and available clinical markers of peripheral inflammation is the neutrophil to lymphocyte ratio (NLR), which has been associated with poor prognosis in several cancer outcomes (Walsh et al., 2005; Sarraf et al., 2009), diabetes (Imtiaz et al., 2012), and cardiovascular disease (CVD) (Bhat et al., 2013). Almost a decade ago, a higher NLR was first reported in patients with AD (Kuyumcu et al., 2012) and replicated in a study using longitudinal data and repetitive measures over time (Rembach et al., 2014). The higher NLR levels observed in patients with $\mathrm{AD}$ were associated with increased amyloid burden, but this association was no longer present after adjustment for age, sex, and ApoE4 status, thus potentially limiting the potential of NLR as a diagnostic biomarker in more advanced stages of the disease. Because of the hyperactive neutrophil state identified in patients with $A D$ and the higher NLR ratios observed in $\mathrm{AD}$ versus controls, it is possible that higher NLR ratios in elderly adults may be independently associated with the risk of incident dementia, although, such an association has yet to be identified.

The aim of this study was to examine whether baseline NLR was independently associated with incident dementia in the Framingham Heart Study (FHS), a longitudinal, communitybased cohort with rigorous and continuous surveillance for clinical dementia incidence and a large number of participants with NLR measures. 


\section{MATERIALS AND METHODS}

\section{Standard Protocol Approvals, Registrations, and Patient Consents}

All participants provided written informed consent. Study protocols and consent forms were approved by the institutional review board at the Boston University Medical Center.

\section{The Framingham Heart Study}

The FHS is one of the few historical active longitudinal cohort studies in the United States, initiated in 1948 and with over 70 years of follow-up evaluations. Following recruitment, the original cohort of 5,209 residents from Framingham, MA, underwent up to 32 examinations every 2 years, where various clinical and laboratory data were collected (Dawber et al., 1963). In 1971, a total of 5,124 children of the original cohort and their spouses were enrolled in the Offspring cohort who completed a total of nine examinations, with the latest examination performed in 2011-2014 (Kannel et al., 1979). Offspring cohort participants who attended the ninth examination cycle (2011-2014), during which a complete blood count (CBC) was obtained, were eligible for the present investigation.

\section{Blood Collection and Complete Blood Count}

Participants were asked to fast after 8:00 p.m., the evening before their clinic exam, and considered to be fasting after a minimum of a 10-h fast. Blood was drawn from participants in a supine position, using standard venipuncture technique, typically between 7:00 a.m. and 9:00 a.m. Hematology testing was performed using whole blood [Tyco Monoject, $15 \%$ ethylenediaminetetraacetic acid, EDTA, (K3)]. A CBC with differential was performed on EDTA whole blood using a Beckman Coulter HmX Hematology Analyzer as described previously (Sloan et al., 2015). Blood collection was performed following the exact same procedures in every subject. Hematology testing included estimation of intra-assay CV in $\sim 10 \%$ samples run in duplicate. The NLR was defined as the absolute neutrophil count by the absolute lymphocyte count from the CBC panel. Assessment of cognitive decline and dementia was performed by a panel blinded to the $\mathrm{CBC} / \mathrm{NLR}$ results.

\section{Dementia Characterization in the Framingham Heart Study}

The main outcome of the present study was an incident clinical diagnosis of dementia using continuous surveillance until the conclusion of the follow-up period up to 2018. The diagnosis of dementia was based on criteria from the Diagnostic and Statistical Manual of Mental Disorders, fourth edition (DSM-IV). The diagnosis of $\mathrm{AD}$ was based on criteria for possible, probable, or definite $\mathrm{AD}$ from the National Institute of Neurological and Communicative Disorders and Stroke and the $\mathrm{AD}$ and Related Disorders Association (NINCDS-ADRDA). Additional methods for continuous surveillance of dementia and $\mathrm{AD}$ in the FHS have been previously described (Seshadri et al., 1997, 2011; Au et al., 2012; Satizabal et al., 2016). In brief, the cognitive function of all participants was assessed using the Mini-Mental State Examination (MMSE) (Folstein et al., 1975) at every examination. The MMSE was used to flag participants for a dementia panel review based on their performance if any of the following occurred: (a) an absolute score $<23$ for all persons, (b) score $<24$ for persons with only high school completed, (c) score $<26$ for participants with a college education, (d) $>3$-point decline between successive examinations, or (e) $>5$-point decline from the highest obtained MMSE scores of the participants. Participants were also flagged for additional evaluation based on concerns from participants themselves, relatives, or other professionals. In additional visits, flagged participants had a full neuropsychological and neurological examination, which was also reviewed to refer for dementia evaluation by the dementia review panel. The panel, which includes neurologists (JS and SS) and neuropsychologists, used data from multiple sources to assess possible cognitive decline and dementia to determine whether a participant had dementia, the dementia subtype, and the date of diagnosis. After a participant died, a medical panel manually reviewed medical records up to the date of death to assess for the potential cognitive decline since the last examination of the participants. This medical panel referred any participants who may have presented with a cognitive decline for postmortem review by the dementia review panel [refer to Satizabal et al. (2016) - Supplementary Material, for a detailed description of dementia surveillance in the FHS]. The main outcome of the present study was incident dementia using continuous surveillance with clinician diagnosis at the conclusion of the follow-up period up to 2018.

\section{Demographic and Clinical Covariates}

Clinic examination corresponding to the $\mathrm{CBC}$ (examination cycle 9) was defined as the baseline. Smoking was defined based on smoking status prior to the year of baseline. We defined diabetes mellitus by fasting glucose levels above $126 \mathrm{mg} / \mathrm{dl}(7.0 \mathrm{mmol} / \mathrm{L})$ and/or use of antidiabetic treatments. Levels of all cardiovascular risk variables, including body mass index (BMI), total cholesterol, high-density lipoprotein (HDL) cholesterol, and triglycerides, were determined from examination cycle 9. History of CVD events at the time of clinical examination included: reported history of coronary heart disease (CHD), congestive heart failure (CHF), myocardial infarct (MI), intermittent claudication (IC), ischemic stroke, intracerebral hemorrhage, or transient ischemic attack (TIA).

\section{Statistical Analysis}

R 3.6 and Python 3.7 were used for statistical analysis and visualization. The analysis involved the following variables: (a) the NLR; (b) outcome: clinical dementia diagnosis; and, (c) basic demographic and clinical parameters (continuous or categorical), which included age, sex, BMI, smoking, HDL, low-density lipoprotein (LDL), total cholesterol, triglycerides, systolic and diastolic blood pressure, diabetes, and history of CVD. Univariate analysis of demographic and clinical differences between study groups included $t$-tests for continuous variables and $\chi^{2}$ tests for nominal variables. Kruskal-Wallis tests were applied to nonnormal distributions. Spearman correlations were used to test the 
association between continuous variables. Generally, we defined statistical significance by $p<0.05$, and tendencies by $p \leq 0.1$.

Follow-up duration was calculated from the date of the $\mathrm{CBC}$ until the most recently available clinical diagnosis prior to the end of follow-up in 2018. To detect potentially independent associations between the NLR and incident dementia, we fit Cox proportional hazard models with inverse probability weighting (IPW). The IPW method weighted each subject by the inverse of the probability of their observed NLR using the median as a cutoff, adjusting for non-random selection of participants into high versus low NLR groups. A logistic regression model for high versus low NLR, adjusted for age, sex, BMI, diabetes, current smoking status, LDH, LDL, total cholesterol, triglycerides, systolic and diastolic blood pressure, and history of CVD was used to estimate these probabilities. The Cox models were then weighted by the inverse of the probabilities of the NLR, adjusted for the above covariates. The proportional hazards assumption was validated using the Schoenfeld residual test included in the cox.zph function of the coxph package (Grambsch, 1995; In and Lee, 2019). Next, using the propensity score model for low versus high NLR (split at the median) for all participants and the causalCmprsk package (Vakulenko-Lagun et al., 2020), we estimated the cumulative incidence function (CIF) for dementia for the two groups. Due to the competing risk of death, the models can be interpreted as cause-specific hazard models (i.e., for the risk of dementia among those still alive). Finally, in an exploratory approach, we applied the survival data implementation of Breiman's random forest models in the randomForestSRC (O'Brien et al., 2021) to estimate the relative importance of each of the covariates in dementia risk. Briefly, the variable importance of each predictor is estimated by using variable selection methods of random forest survival models. The variable selection method uses a prediction error approach by "noising-up" each variable in turn. The variable importance of a variable $\mathrm{X}_{\mathrm{i}}$ is the difference in prediction error when $\mathrm{X}_{\mathrm{i}}$ is randomly permuted, compared to the prediction error under the true values. The package ggRandomForests was used for visualization. Code is available upon reasonable request and for collaboration and reproducibility purposes. The data are available in the BioLINCC repositories.

\section{RESULTS}

\section{Study Sample}

The final study sample consisted of 1,648 FHS participants and included the combination of NLR and clinical and demographic covariates (Figure 1) described previously. Table 1 summarizes baseline characteristics for all participants at the time of the CBC. The average age of participants in the study at baseline was 69 years [the interquartile range (IQR): 64, 76]; 55.8\% were female (920).

\section{Neutrophil to Lymphocyte Ratio}

Intra-assay CVs were $<2.5 \%$ for both the total neutrophil and lymphocyte counts. The absolute neutrophil and lymphocyte count and the NLR followed a unimodal distribution.
Although the participants with a diagnosis of dementia at baseline were excluded from the analysis, a brief comparison of the clinical labs at baseline indicated higher neutrophil counts and NLR in dementia cases (Supplementary Figure 1). In the final cohort (non-dementia) participants that showed a higher NLR (above median) were older and predominantly male; they had lower LDL, HDL, and total cholesterol and higher BMI, triglycerides, systolic blood pressure, and rates of CVD history at baseline $(p<0.05$; Table 1$)$.

\section{Association of the Neutrophil to Lymphocyte Ratio and Incident Dementia}

Of the 1,648 participants, 51 cases of incident dementia (41 confirmed $\mathrm{AD}$ cases) and 85 deaths without incident dementia were observed during a median follow-up of 5.9 years (IQR: 2.6, 6.9). Logistic models used to estimate IPW weights indicated strong associations between the NLR and age, male sex, BMI, and smoking status (Supplementary Table 1). Although the CIs are overlapping, and there is insufficient power to conclude that the incidence curves are different, CIF curves suggested that groups with a higher baseline NLR demonstrated a greater incidence of dementia (Figure 2). For example, the probability of dementia prior to death occurring within 5.9 years for those with low NLR at baseline is $2.73 \%$ [95\% CI: $1.56,4.43$ ], while for those with high NLR it was $4.11 \%$ [95\% CI: 3.05, 5.38]. Ranked feature importance of random forest competing for risk models assigned highest priority to age, systolic and diastolic blood pressure, and history of CVD, immediately followed by the NLR (Figure 3). Variables with lower importance than the NLR included total cholesterol, diabetes, BMI, LDL cholesterol, smoking status, sex, and triglycerides. Adjusted hazard ratios (HRs) for the IPW multivariate Cox proportional hazard models for incident dementia are summarized in Supplementary Tables 2, 3. Higher NLR at baseline was independently associated with incident dementia when included as a continuous variable (HR: 1.22; 95\% CI: $1.05,1.43 ; p=0.01)$. Results indicated a $22 \%$ increase in dementia risk per unit increase in the NLR. Elevated HRs are

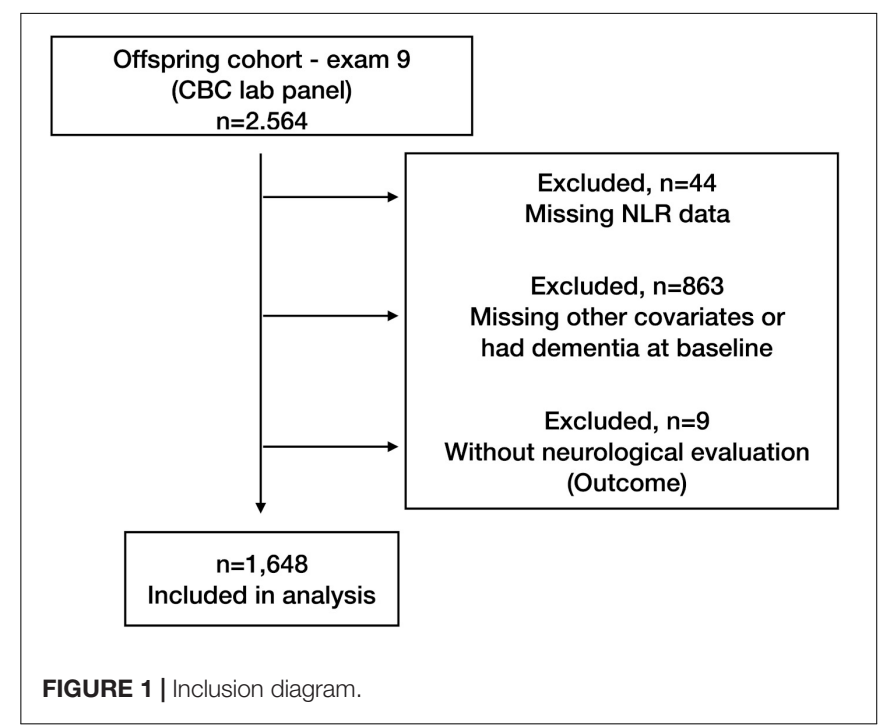


TABLE 1 | Comparison of demographic and clinical characteristics by the neutrophil to lymphocyte ratio (NLR).

\begin{tabular}{|c|c|c|c|c|}
\hline & Overall $(n=1,648)$ & NLR below median ( $n=826$ ) & NLR above median ( $n=822)$ & $P$-value \\
\hline Age, median [Q1, Q3] & $69.0[64.0,76.0]$ & $68.0[63.0,74.0]$ & $71.0[65.0,77.0]$ & $<0.001$ \\
\hline Female sex, $n(\%)$ & $920(55.8)$ & $515(62.3)$ & 405 (49.3) & $<0.001$ \\
\hline BMI, median [Q1, Q3] & $27.7[24.7,30.9]$ & $27.5[24.3,30.5]$ & $28.0[25.1,31.4]$ & 0.006 \\
\hline LDL cholesterol, median [Q1, Q3] & $96.0[78.0,118.0]$ & $101.5[81.0,122.0]$ & $93.0[75.0,112.0]$ & $<0.001$ \\
\hline HDL cholesterol, median [Q1, Q3] & $60.0[49.0,74.0]$ & $61.0[50.0,76.0]$ & $58.0[47.0,71.0]$ & $<0.001$ \\
\hline Total cholesterol, median [Q1, Q3] & $183.0[158.8,207.0]$ & $188.0[163.2,211.0]$ & $177.0[153.0,202.0]$ & $<0.001$ \\
\hline Triglycerides, median [Q1, Q3] & $99.0[74.0,133.0]$ & $96.0[72.0,132.0]$ & $100.5[76.0,134.0]$ & 0.041 \\
\hline Smoker, $n(\%)$ & $78(4.7)$ & $32(3.9)$ & $46(5.6)$ & 0.126 \\
\hline Diabetes, $n(\%)$ & $159(9.6)$ & $76(9.2)$ & $83(10.1)$ & 0.594 \\
\hline Systolic BP, median [Q1, Q3] & $125.0[115.0,137.0]$ & $125.0[113.0,136.0]$ & $126.0[116.0,137.0]$ & 0.011 \\
\hline Diastolic BP, median [Q1, Q3] & $72.0[65.0,78.0]$ & $72.0[66.0,78.0]$ & $71.0[65.0,78.0]$ & 0.176 \\
\hline CVD History, $n$ (\%) & 295 (17.9) & $122(14.8)$ & $173(21.0)$ & 0.001 \\
\hline NLR, median [Q1, Q3] & $2.3[1.8,3.0]$ & $1.8[1.4,2.0]$ & $3.0[2.6,3.8]$ & $<0.001$ \\
\hline Number of dementia cases, $n(\%)$ & $51(3.1)$ & $16(1.9)$ & $35(4.3)$ & 0.01 \\
\hline Number of deaths, $n(\%)$ & $92(5.6)$ & $33(4.0)$ & $59(7.2)$ & 0.007 \\
\hline
\end{tabular}

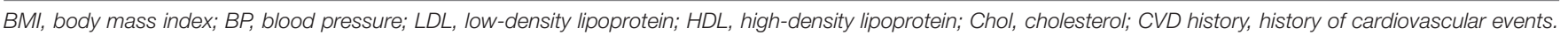
Definitions described in section "Materials and Methods."

also apparent with respect to categorical coding of NLR: above versus below the median (HR: 1.80 ; 95\% CI: $0.96,3.37 ; p=0.07$ ) in adjusted models. A sensibility analysis inspecting confirmed $\mathrm{AD}$ cases as an outcome similarly suggested elevated NLR was associated with higher rates of incident AD (Supplementary Tables 4, 5 and Supplementary Figure 2).

\section{DISCUSSION}

Recently, neutrophils have been identified as potential innate immunity players contributing to $\mathrm{AD}$ pathology, and a hyperactive neutrophil state has been found in patients with $\mathrm{AD}$ and associated with $\mathrm{AD}$ progression. The NLR serves as a composite inflammatory biomarker, which incorporates information from two leukocyte subtypes. Exploration of the NLR as a proxy for neutrophil-mediated inflammation is of interest since the NLR can be obtained as part of the standard CBC panel, which is widely available in most hospitals. Our study in the community-based longitudinal FHS Offspring cohort demonstrated that individuals with elevated NLR are at a greater risk of dementia during a 5.9-year follow-up period. These findings persist after adjustment for a number of covariates that could play a confounding role in the association of NLR with dementia risk. Importantly, results from the sensitivity analysis suggested an association of the NLR and AD risk specifically.

The role of neutrophils in $\mathrm{AD}$ pathology and clinical progression has been previously suggested (Shad et al., 2013; Pietronigro et al., 2017; Dong et al., 2018; Stock et al., 2018; Bawa et al., 2020; Rossi et al., 2021). Although variations in the NLR have been associated with several clinical outcomes other than AD (Walsh et al., 2005; Sarraf et al., 2009; Imtiaz et al., 2012; Bhat et al., 2013), the interpretation of a change in the NLR remains controversial. Studies in animal models suggest that clearance of neutrophils may be reduced with aging, potentially compromising inflammation resolution following injury. The increased NLR with aging in humans may be indicative of such a process and whether abnormal neutrophil phenotypes are associated with the increased NLR should be studied in the future. Recent studies show that neutrophils may be involved in capillary stall formation and contribute to $\mathrm{CBF}$ reductions that when sustained over time may lead to oxidative stress, endothelial damage and influence the pathological accumulation of $A \beta$ and

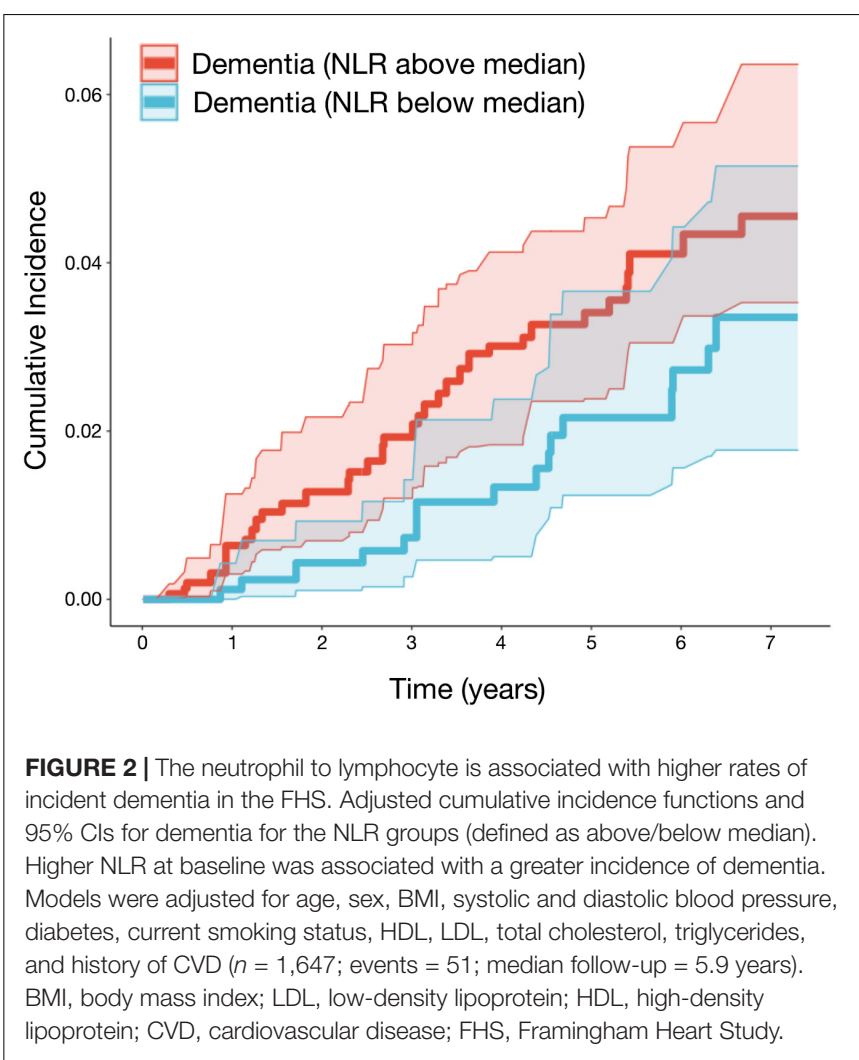




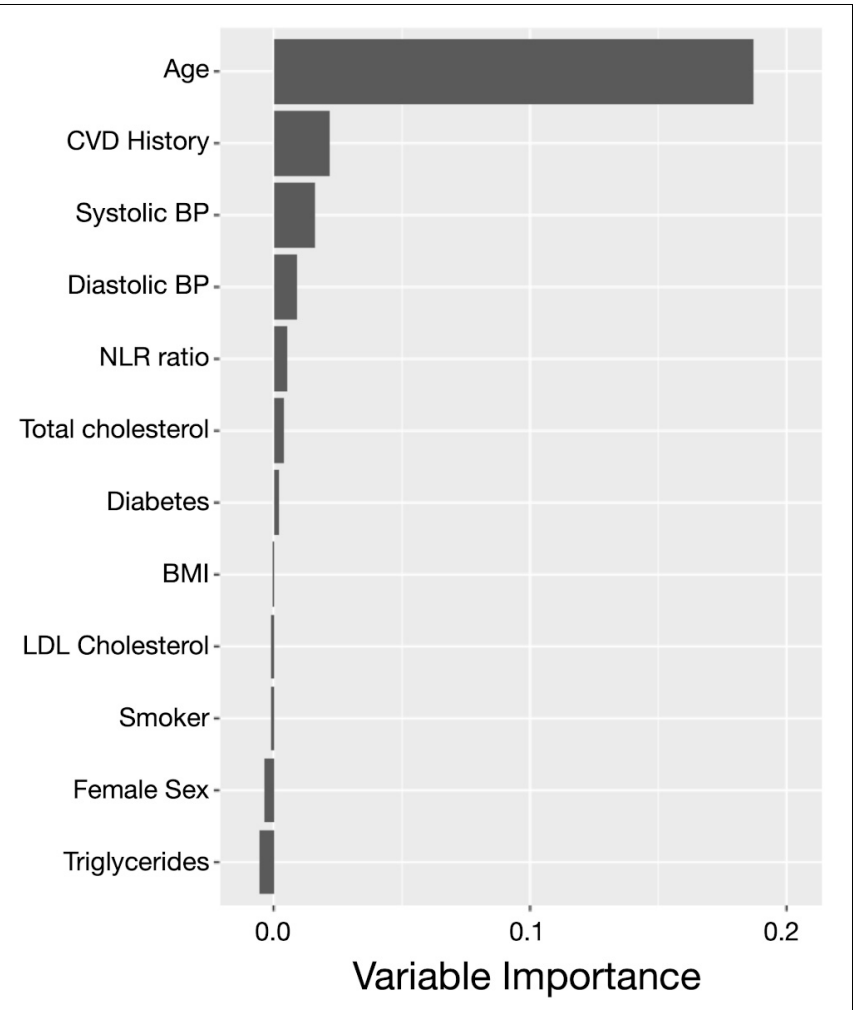

FIGURE 3 | The neutrophil to lymphocyte ratio plays an important role in incident dementia prediction. Ranked feature importance for random forest survival models for the prediction of incident dementia assigned a higher priority for the NLR (coded as continuous) over other clinical covariates. The highest priority was given to age, history of CVD, and hypertension, followed by the NLR and the remaining clinical and demographic covariates. Variable importance close to zero indicates the variable contributed nothing to predictive accuracy, and negative values indicate the predictive accuracy improved when the variable was misspecified and therefore was not informative. CVD, cardiovascular disease; FHS, Framingham Heart Study; NLR, neutrophil to lymphocyte ratio. tau at the early stages of the disease (Cruz Hernandez et al., 2019; El Amki et al., 2020). Additionally, the vascular dysfunction state that is associated with $\mathrm{AD}$ may increase endothelial signaling facilitating neutrophil recruitment to the capillary vessel walls in a feed-forward loop. Studies need to evaluate whether the stall formations are associated with increased NLR.

Although our primary goal was to evaluate the association of the NLR with incident dementia, we also observed higher NLR in the dementia cases at baseline that was excluded from analysis when compared to dementia-free participants. The results are in agreement with previous research that identified higher NLR in patients with $\mathrm{AD}$ when compared to age-matched controls (Kuyumcu et al., 2012; Rembach et al., 2014). The NLR ratio has also been associated with conditions related to AD risk and influencing progressions, such as CVD (Angkananard et al., 2018; Kim et al., 2018; Haybar et al., 2019), obstructive sleep apnea (Altintas et al., 2015; Sunbul et al., 2015; Oyama et al., 2016; Rha et al., 2020), depression (Aydin Sunbul et al., 2016; Demircan et al., 2016; Arabska et al., 2018; Liang et al., 2019), and obesity
(Rodriguez-Rodriguez et al., 2020). Our findings are therefore consistent with previous research that identified an association of the NLR with AD and related risk factors.

In observational studies like the FHS where there is no random assignment to treatment groups (or variable of interest like the NLR in this case), the unadjusted comparison between treatment groups may be misleading due to confounding. To adjust for measured confounders in our study, we used inverse probability of treatment weighting which is a robust approach for correcting for potential confounding (Neumann and Billionnet, 2016; Vakulenko-Lagun et al., 2020). In our study using weighted Cox models in elderly adults and a 5.9-year median followup suggested that the NLR was independently associated with incident dementia. Additionally, the variable importance results of random forest survival models suggested that the NLR had a considerable high predictive value when compared to other variables known to be relevant in dementia risks, such as age, sex, BMI, blood pressure, diabetes, smoking status, lipids, and history of CVD, suggesting that the NLR may add predictive value when incorporated in machine-learning models for dementia and this needs to be examined in future studies.

Our study has several limitations. First, although we adjusted for a relatively large number of covariates, we did not exclude/adjust for psychiatric and autoimmune disorders known to increase dementia risk that also to may influence the NLR ratios (e.g., depression) and future analysis should control for these conditions and look at each of the risk groups separately. Additionally, the number of dementia cases available for risk stratification in our study was relatively small and future studies should replicate our analysis if a larger number of incipient dementia cases are available. Second, in our primary analysis, we did not control for the number of years of education, the use of hypertensive drugs, and drugs potentially modulating the $\mathrm{CBC}$ results due to a considerable number of missing observations that limited the number of incipient dementia cases available for analysis. A sensitivity analysis also including the aforementioned variables showed associations with the same directionality of NLR associated with increased risk of incipient dementia. Additionally, our study did not account for the ApoE4 status, which is the main genetic risk factor for AD (Tsai et al., 1994; Kim et al., 2009; Liu et al., 2013) and previous research found that the ApoE4 status influences the NLR (Rembach et al., 2014). Cholesterol homeostasis has a pivotal function in regulating immune cells, and the role of ApoE in neutrophilmediated inflammation has been suggested in experimental models (Terkeltaub et al., 1991; Barger and Harmon, 1997; Zhou et al., 2019; Doring et al., 2020).

\section{CONCLUSION}

Our study in 1,648 participants of a well-characterized community-based cohort shows an independent association of the NLR with future dementia, reinforcing the role of neutrophil-mediated inflammation in $\mathrm{AD}$ pathology and progression. 


\section{FUTURE PERSPECTIVES}

Future studies need to determine whether the increased NLR observed in certain participants at higher risk of dementia is independent or mediated by the ApoE4 status and the role of other AD-risk factors in the association. Additionally, dementia is more frequent in women and some race and ethnic groups, and we were not powered to perform disaggregated analyses. Finally, whether the NLR is associated with other markers of vulnerability to cognitive decline (global cognition, MRI, PET, and ADbiofluid markers) in middle age needs to be further investigated.

\section{DATA AVAILABILITY STATEMENT}

Publicly available datasets were analyzed in this study. This data can be found here: https://biolincc.nhlbi.nih.gov/home/.

\section{ETHICS STATEMENT}

The studies involving human participants were reviewed and approved by the Boston University Medical Center. The patients/participants provided their written informed consent to participate in this study.

\section{AUTHOR CONTRIBUTIONS}

JR-C designed and conceptualized the study, analyzed the data, performed the statistical analysis, and drafted the manuscript. AJ, $\mathrm{AB}, \mathrm{SS}$, and JS interpreted the data, major role in the acquisition of data, and revised the manuscript for intellectual content. JB, $\mathrm{NF}, \mathrm{ND}, \mathrm{CZ}, \mathrm{ZK}, \mathrm{OB}, \mathrm{AP}, \mathrm{AC}, \mathrm{RB}$, and TW revised the manuscript

\section{REFERENCES}

Altintas, N., Cetinoglu, E., Yuceege, M., Acet, A. N., Ursavas, A., Firat, H., et al. (2015). Neutrophil-to-lymphocyte ratio in obstructive sleep apnea; a multi center, retrospective study. Eur. Rev. Med. Pharmacol. Sci. 19, 3234-3240.

Angkananard, T., Anothaisintawee, T., McEvoy, M., Attia, J., and Thakkinstian, A. (2018). Neutrophil lymphocyte ratio and cardiovascular disease risk: a systematic review and meta-analysis. Biomed. Res. Int. 2018:2703518. doi: 10. 1155/2018/2703518

Arabska, J., Lucka, A., Magierski, R., Sobow, T., and Wysokinski, A. (2018). Neutrophil-lymphocyte ratio is increased in elderly patients with first episode depression, but not in recurrent depression. Psychiatry Res. 263, 35-40. doi: 10.1016/j.psychres.2018.02.043

Au, R., Seshadri, S., Knox, K., Beiser, A., Himali, J. J., Cabral, H. J., et al. (2012). The Framingham brain donation program: neuropathology along the cognitive continuum. Curr. Alzheimer Res. 9, 673-686. doi: 10.2174/ 156720512801322609

Aydin Sunbul, E., Sunbul, M., Yanartas, O., Cengiz, F., Bozbay, M., Sari, I., et al. (2016). Increased neutrophil/lymphocyte ratio in patients with depression is correlated with the severity of depression and cardiovascular risk factors. Psychiatry Investig. 13, 121-126. doi: 10.4306/pi.2016.13.1.121

Baik, S. H., Cha, M. Y., Hyun, Y. M., Cho, H., Hamza, B., Kim, D. K., et al. (2014). Migration of neutrophils targeting amyloid plaques in Alzheimer's disease mouse model. Neurobiol. Aging 35, 1286-1292. doi: 10.1016/j.neurobiolaging. 2014.01.003 for intellectual content. TW and RO designed and conceptualized study, interpreted the results, and revised the manuscript for intellectual content. All authors contributed to the article and approved the submitted version.

\section{FUNDING}

This work presented here was supported by the NIH funding from RO (R01AG056531 and R01AG056031) and JB (NCT02106429 and NCT01897103). JR-C was supported by an Intergovernmental Personnel Act with the VA Boston Healthcare System (Cooperative Studies Program). AJ was supported by NHLBI Intramural funds. TW, RB, and RO were supported by P30 AG066512. JS was supported by K23AG057760. AP was funded by NIH K25HL151912. OB was supported by the National Institute on Aging (K23AG068534 and L30-AG064670) and American Academy of Sleep Medicine Foundation (BS-23120). AC was supported by R01 AG055624. The Framingham Heart Study was funded by contract 75N92019D00031 and Dementia grant AG054076.

\section{ACKNOWLEDGMENTS}

We thank the participants of the Framingham Heart Study.

\section{SUPPLEMENTARY MATERIAL}

The Supplementary Material for this article can be found online at: https://www.frontiersin.org/articles/10.3389/fnagi. 2021.773984/full\#supplementary-material

Barger, S. W., and Harmon, A. D. (1997). Microglial activation by Alzheimer amyloid precursor protein and modulation by apolipoprotein E. Nature 388, 878-881. doi: 10.1038/42257

Bawa, K. K., Krance, S. H., Herrmann, N., Cogo-Moreira, H., Ouk, M., Yu, D., et al. (2020). A peripheral neutrophil-related inflammatory factor predicts a decline in executive function in mild Alzheimer's disease. J. Neuroinflammation 17:84. doi: 10.1186/s12974-020-01750-3

Bhat, T., Teli, S., Rijal, J., Bhat, H., Raza, M., Khoueiry, G., et al. (2013). Neutrophil to lymphocyte ratio and cardiovascular diseases: a review. Expert. Rev. Cardiovasc. Ther. 11, 55-59. doi: 10.1586/erc.12.159

Cruz Hernandez, J. C., Bracko, O., Kersbergen, C. J., Muse, V., Haft-Javaherian, M., Berg, M., et al. (2019). Neutrophil adhesion in brain capillaries reduces cortical blood flow and impairs memory function in Alzheimer's disease mouse models. Nat. Neurosci. 22, 413-420. doi: 10.1038/s41593-018-03 29-4

Cunningham, C., and Hennessy, E. (2015). Co-morbidity and systemic inflammation as drivers of cognitive decline: new experimental models adopting a broader paradigm in dementia research. Alzheimers Res. Ther. 7:33. doi: 10.1186/s13195-015-0117-2

Dawber, T. R., Kannel, W. B., and Lyell, L. P. (1963). An approach to longitudinal studies in a community: the Framingham study. Ann. N. Y. Acad. Sci. 107, 539-556. doi: 10.1111/j.1749-6632.1963.tb13299.x

Demircan, F., Gozel, N., Kilinc, F., Ulu, R., and Atmaca, M. (2016). The impact of red blood cell distribution width and neutrophil/lymphocyte ratio on the diagnosis of major depressive disorder. Neurol. Ther. 5, 27-33. doi: 10.1007/ s40120-015-0039-8 
Dong, Y., Lagarde, J., Xicota, L., Corne, H., Chantran, Y., Chaigneau, T., et al. (2018). Neutrophil hyperactivation correlates with Alzheimer's disease progression. Ann. Neurol. 83, 387-405. doi: 10.1002/ana.25159

Doring, Y., Libby, P., and Soehnlein, O. (2020). Neutrophil extracellular traps participate in cardiovascular diseases: recent experimental and clinical insights. Circ. Res. 126, 1228-1241. doi: 10.1161/CIRCRESAHA.120.31 5931

El Amki, M., Gluck, C., Binder, N., Middleham, W., Wyss, M. T., Weiss, T., et al. (2020). Neutrophils obstructing brain capillaries are a major cause of no-reflow in ischemic stroke. Cell Rep. 33:108260. doi: 10.1016/j.celrep.2020.108260

Folstein, M. F., Folstein, S. E., and McHugh, P. R. (1975). "Mini-mental state”. A practical method for grading the cognitive state of patients for the clinician. J. Psychiatr. Res. 12, 189-198. doi: 10.1016/0022-3956(75)90026-6

Grambsch, P. M. (1995). Goodness-of-fit and diagnostics for proportional hazards regression models. Cancer Treat. Res. 75, 95-112. doi: 10.1007/978-1-46152009-2_5

Haybar, H., Pezeshki, S. M. S., and Saki, N. (2019). Evaluation of complete blood count parameters in cardiovascular diseases: an early indicator of prognosis? Exp. Mol. Pathol. 110:104267. doi: 10.1016/j.yexmp.2019.10 4267

Holmes, C., Cunningham, C., Zotova, E., Woolford, J., Dean, C., Kerr, S., et al. (2009). Systemic inflammation and disease progression in Alzheimer disease. Neurology 73, 768-774. doi: 10.1212/WNL.0b013e3181b6bb95

Imtiaz, F., Shafique, K., Mirza, S. S., Ayoob, Z., Vart, P., and Rao, S. (2012). Neutrophil lymphocyte ratio as a measure of systemic inflammation in prevalent chronic diseases in Asian population. Int. Arch. Med. 5:2. doi: 10. $1186 / 1755-7682-5-2$

In, J., and Lee, D. K. (2019). Survival analysis: part II - applied clinical data analysis. Korean J. Anesthesiol. 72, 441-457. doi: 10.4097/kja.19183

Iturria-Medina, Y., Sotero, R. C., Toussaint, P. J., Mateos-Perez, J. M., Evans, A. C., Weiner, M. W., et al. (2016). Early role of vascular dysregulation on late-onset Alzheimer's disease based on multifactorial data-driven analysis. Nat. Commun. 7:11934. doi: 10.1038/ncomms11934

Kannel, W. B., Feinleib, M., McNamara, P. M., Garrison, R. J., and Castelli, W. P. (1979). An investigation of coronary heart disease in families. The Framingham offspring study. Am. J. Epidemiol. 110, 281-290. doi: 10.1093/oxfordjournals. aje.a112813

Kim, J., Basak, J. M., and Holtzman, D. M. (2009). The role of apolipoprotein E in Alzheimer's disease. Neuron 63, 287-303. doi: 10.1016/j.neuron.2009.06.026

Kim, S., Eliot, M., Koestler, D. C., Wu, W. C., and Kelsey, K. T. (2018). Association of neutrophil-to-lymphocyte ratio with mortality and cardiovascular disease in the Jackson Heart Study and modification by the Duffy Antigen variant. JAMA Cardiol. 3, 455-462. doi: 10.1001/jamacardio.2018.1042

Kruger, P., Saffarzadeh, M., Weber, A. N., Rieber, N., Radsak, M., von Bernuth, H., et al. (2015). Neutrophils: between host defence, immune modulation, and tissue injury. PLoS Pathog. 11:e1004651. doi: 10.1371/journal.ppat.100 4651

Kuyumcu, M. E., Yesil, Y., Ozturk, Z. A., Kizilarslanoglu, C., Etgul, S., Halil, M., et al. (2012). The evaluation of neutrophil-lymphocyte ratio in Alzheimer's disease. Dement. Geriatr. Cogn. Disord. 34, 69-74. doi: 10.1159/00034 1583

Liang, M., Du, B., Zhang, H., Lu, X., Chen, C., Fan, C., et al. (2019). NLR is associated with geriatric depression in chinese women: a community-based cross-sectional study in Eastern China. Front. Psychol. 10:2941. doi: 10.3389/ fpsyg.2019.02941

Liu, C. C., Liu, C. C., Kanekiyo, T., Xu, H., and Bu, G. (2013). Apolipoprotein E and Alzheimer disease: risk, mechanisms and therapy. Nat. Rev. Neurol. 9, 106-118. doi: $10.1038 /$ nrneurol.2012.263

Love, S., and Miners, J. S. (2016). Cerebrovascular disease in ageing and Alzheimer's disease. Acta Neuropathol. 131, 645-658. doi: 10.1007/s00401-015-1522-0

Nemeth, T., Sperandio, M., and Mocsai, A. (2020). Neutrophils as emerging therapeutic targets. Nat. Rev. Drug Discov. 19, 253-275. doi: 10.1038/s41573019-0054-Z

Neumann, A., and Billionnet, C. (2016). Covariate adjustment of cumulative incidence functions for competing risks data using inverse probability of treatment weighting. Comput. Methods Programs Biomed. 129, 63-70. doi: 10. 1016/j.cmpb.2016.03.008
Nortley, R., Korte, N., Izquierdo, P., Hirunpattarasilp, C., Mishra, A., Jaunmuktane, Z., et al. (2019). Amyloid beta oligomers constrict human capillaries in Alzheimer's disease via signaling to pericytes. Science 365:eaav9518. doi: 10. 1126/science.aav9518

O’Brien, R. C., Ishwaran, H., Szczotka-Flynn, L. B., Lass, J. H., and Cornea Preservation Time Study (2021). Random survival forests analysis of intraoperative complications as predictors of descemet stripping automated endothelial Keratoplasty graft failure in the cornea preservation time study. JAMA Ophthalmol. 139, 191-197. doi: 10.1001/jamaophthalmol.2020.5743

Oyama, J., Nagatomo, D., Yoshioka, G., Yamasaki, A., Kodama, K., Sato, M., et al. (2016). The relationship between neutrophil to lymphocyte ratio, endothelial function, and severity in patients with obstructive sleep apnea. J. Cardiol. 67, 295-302. doi: 10.1016/j.jjcc.2015.06.005

Pietronigro, E. C., Della Bianca, V., Zenaro, E., and Constantin, G. (2017). NETosis in Alzheimer's Disease. Front. Immunol. 8:211. doi: 10.3389/fimmu.2017. 00211

Pillay, J., den Braber, I., Vrisekoop, N., Kwast, L. M., de Boer, R. J., Borghans, J. A., et al. (2010). In vivo labeling with $2 \mathrm{H} 2 \mathrm{O}$ reveals a human neutrophil lifespan of 5.4 days. Blood 116, 625-627. doi: 10.1182/blood-2010-01-259028

Rembach, A., Watt, A. D., Wilson, W. J., Rainey-Smith, S., Ellis, K. A., Rowe, C. C., et al. (2014). An increased neutrophil-lymphocyte ratio in Alzheimer's disease is a function of age and is weakly correlated with neocortical amyloid accumulation. J. Neuroimmunol. 273, 65-71. doi: 10.1016/j.jneuroim.2014.05. 005

Rha, M. S., Kim, C. H., Yoon, J. H., and Cho, H. J. (2020). Association between the neutrophil-to-lymphocyte ratio and obstructive sleep apnea: a meta-analysis. Sci. Rep. 10:10862. doi: 10.1038/s41598-020-67708-w

Rodriguez-Rodriguez, E., Lopez-Sobaler, A. M., Ortega, R. M., Delgado-Losada, M. L., Lopez-Parra, A. M., and Aparicio, A. (2020). Association between neutrophil-to-lymphocyte ratio with abdominal obesity and healthy eating index in a representative older Spanish Population. Nutrients 12:855. doi: 10. 3390/nu12030855

Rossi, B., Santos-Lima, B., Terrabuio, E., Zenaro, E., and Constantin, G. (2021). Common peripheral immunity mechanisms in multiple sclerosis and Alzheimer's disease. Front. Immunol. 12:639369. doi: 10.3389/fimmu.2021. 639369

Sarraf, K. M., Belcher, E., Raevsky, E., Nicholson, A. G., Goldstraw, P., and Lim, E. (2009). Neutrophil/lymphocyte ratio and its association with survival after complete resection in non-small cell lung cancer. J. Thorac. Cardiovasc. Surg. 137, 425-428. doi: 10.1016/j.jtcvs.2008.05.046

Satizabal, C. L., Beiser, A. S., Chouraki, V., Chene, G., Dufouil, C., and Seshadri, S. (2016). Incidence of dementia over three decades in the Framingham heart study. N. Engl. J. Med. 374, 523-532. doi: 10.1056/NEJMoa1504327

Seshadri, S., Beiser, A., Au, R., Wolf, P. A., Evans, D. A., Wilson, R. S., et al. (2011). Operationalizing diagnostic criteria for Alzheimer's disease and other age-related cognitive impairment-Part 2. Alzheimers Dement. 7, 35-52. doi: 10.1016/j.jalz.2010.12.002

Seshadri, S., Wolf, P. A., Beiser, A., Au, R., McNulty, K., White, R., et al. (1997). Lifetime risk of dementia and Alzheimer's disease. The impact of mortality on risk estimates in the Framingham Study. Neurology 49, 1498-1504. doi: 10.1212/wnl.49.6.1498

Shad, K. F., Aghazadeh, Y., Ahmad, S., and Kress, B. (2013). Peripheral markers of Alzheimer's disease: surveillance of white blood cells. Synapse 67, 541-543. doi: 10.1002/syn.21651

Sloan, A., Gona, P., and Johnson, A. D. (2015). Cardiovascular correlates of platelet count and volume in the Framingham heart study. Ann. Epidemiol. 25, 492-498. doi: 10.1016/j.annepidem.2015.01.010

Stock, A. J., Kasus-Jacobi, A., and Pereira, H. A. (2018). The role of neutrophil granule proteins in neuroinflammation and Alzheimer's disease. J. Neuroinflammation 15:240. doi: 10.1186/s12974-018-1284-4

Sunbul, M., Sunbul, E. A., Kanar, B., Yanartas, O., Aydin, S., Bacak, A., et al. (2015). The association of neutrophil to lymphocyte ratio with presence and severity of obstructive sleep apnea. Bratisl. Lek. Listy 116, 654-658. doi: 10.4149/bll_2015_ 125

Terkeltaub, R. A., Dyer, C. A., Martin, J., and Curtiss, L. K. (1991). Apolipoprotein (apo) E inhibits the capacity of monosodium urate crystals to stimulate neutrophils. Characterization of intraarticular apo $\mathrm{E}$ and demonstration of apo 
E binding to urate crystals in vivo. J. Clin. Invest. 87, 20-26. doi: 10.1172/ JCI114971

Tsai, M. S., Tangalos, E. G., Petersen, R. C., Smith, G. E., Schaid, D. J., Kokmen, E., et al. (1994). Apolipoprotein E: risk factor for Alzheimer disease. Am. J. Hum. Genet. 54, 643-649.

Tseng, C. W., and Liu, G. Y. (2014). Expanding roles of neutrophils in aging hosts. Curr. Opin. Immunol. 29, 43-48. doi: 10.1016/j.coi.2014.03. 009

Vakulenko-Lagun, B., Mandel, M., and Betensky, R. A. (2020). Inverse probability weighting methods for Cox regression with right-truncated data. Biometrics 76, 484-495. doi: 10.1111/biom.13162

Walsh, S. R., Cook, E. J., Goulder, F., Justin, T. A., and Keeling, N. J. (2005). Neutrophil-lymphocyte ratio as a prognostic factor in colorectal cancer. J. Surg. Oncol. 91, 181-184. doi: 10.1002/jso.20329

Zenaro, E., Pietronigro, E., Della Bianca, V., Piacentino, G., Marongiu, L., Budui, S., et al. (2015). Neutrophils promote Alzheimer's disease-like pathology and cognitive decline via LFA-1 integrin. Nat. Med. 21, 880-886. doi: 10.1038/nm. 3913

Zhou, Z., Zhang, S., Ding, S., Abudupataer, M., Zhang, Z., Zhu, X., et al. (2019). Excessive neutrophil extracellular trap formation aggravates acute myocardial infarction injury in Apolipoprotein E deficiency mice via the ROS-dependent pathway. Oxid. Med. Cell Longev. 2019:1209307. doi: 10.1155/2019/120 9307
Author Disclaimer: The views expressed in this manuscript are those of the authors and do not necessarily represent the views of the National Heart, Lung, and Blood Institute, National Institutes of Health, or United States Department of Health and Human Services.

Conflict of Interest: The authors declare that the research was conducted in the absence of any commercial or financial relationships that could be construed as a potential conflict of interest.

Publisher's Note: All claims expressed in this article are solely those of the authors and do not necessarily represent those of their affiliated organizations, or those of the publisher, the editors and the reviewers. Any product that may be evaluated in this article, or claim that may be made by its manufacturer, is not guaranteed or endorsed by the publisher.

Copyright (c) 2021 Ramos-Cejudo, Johnson, Beiser, Seshadri, Salinas, Berger, Fillmore, Do, Zheng, Kovbasyuk, Ardekani, Bubu, Parekh, Convit, Betensky, Wisniewski and Osorio. This is an open-access article distributed under the terms of the Creative Commons Attribution License (CC BY). The use, distribution or reproduction in other forums is permitted, provided the original author(s) and the copyright owner(s) are credited and that the original publication in this journal is cited, in accordance with accepted academic practice. No use, distribution or reproduction is permitted which does not comply with these terms. 